
http://dx.doi.org/10.12819/2021.18.9.1

\title{
Gestão de Suprimentos: Evidenciação e Análise das Publicações do Tema
}

\section{Purchasing Management: Disclosure and Analysis of Publications of the Theme}

Isis Toledo Alexandre

Mestre em Engenharia de Produção pela Universidade Federal de Santa Catarina

E-mail: isis_toledo@hotmail.com

Sandra Rolim Ensslin

Doutora em Engenharia de Produção pela Universidade Federal de Santa Catarina Professora do Programa de Pós-Graduação em Contabilidade da Universidade Federal de Santa Catarina

E-mail: sensslin@gmail.com

Leonardo Corrêa Chaves

Doutor em Administração pela Universidade Federal de Santa Catarina Professor do Programa de Mestrado Profissional em Administração da Universidade do Contestado.

E-mail: leonardomg@gmail.com

João Manuel Dias da Silva

Doutor em Ciências Econômicas pela Universidade do Oriente E-mail: joaod62@gmail.com

Endereço: Isis Toledo Alexandre

R. Eng. Agronômico Andrei Cristian Ferreira, s/n Trindade, Florianópolis - SC, 88040-900. Brasil.

Endereço: Sandra Rolim Ensslin

R. Eng. Agronômico Andrei Cristian Ferreira, s/n Trindade, Florianópolis - SC, 88040-900. Brasil.

Endereço: Leonardo Corrêa Chaves

Av. Presidente, Av. Nereu Ramos, 1071 - Jardim do Moinho, Mafra - SC, 89300-000. Brasil.

Endereço: João Manuel Dias da Silva

Av. Cidade de Lisboa, CP 88, Edifício da ARES, $4^{\circ}$ Piso 7942-056 Várzea, Praia - Santiago, Cabo Verde.
Editor-Chefe: Dr. Tonny Kerley de Alencar Rodrigues

Artigo recebido em 17/08/2021. Última versão recebida em 30/08/2021. Aprovado em 31/08/2021.

Avaliado pelo sistema Triple Review: a) Desk Review pelo Editor-Chefe; e b) Double Blind Review (avaliação cega por dois avaliadores da área).

Revisão: Gramatical, Normativa e de Formatação 


\title{
RESUMO
}

No atual patamar competitivo, empresas precisam se reinventar constantemente com o intento de permanecer sustentáveis. Nesse cenário, a inovação e a agilidade para adaptação a novos requisitos de mercado são atributos primordiais. Para o fomento de tais características, a gestão de suprimentos se torna essencial, pois permite abastecer corporações de forma estratégica para o alcance dos objetivos. Nesse sentido, o objetivo deste estudo, de natureza exploratória e descritiva, consistiu em evidenciar um portfólio bibliográfico referente ao tópico gestão de suprimentos e ressaltar variáveis de destaque sobre o assunto de acordo com as delimitações postas pelos pesquisadores. Para tanto, utilizou-se o Knowledge Development Process - Constructivist (Proknow-C). Obtiveram-se 20 publicações sobre o tema e para esse material concluiu-se que: o Journal of Purchasing and Supply Management é o periódico com maior quantidade de publicações relacionadas; o artigo intitulado como Supply management orientation and supplier/buyer performance destacou-se quanto ao número de citações; os autores que detiveram mais artigos no portfólio bibliográfico são Sánchez-Rodríguez, E. Hartmann e R. Moser e os autores do portfólio bibliográfico que mais foram citados são D. A. Collier e D. D. Wilson; as palavras-chave mais encontradas no portfólio foram performance, seguida por purchasing e management.

Palavras-chave: Suprimentos. Avaliação do Desempenho. Organizações. Proknow-C.

\begin{abstract}
In today's competitive environment, companies need to constantly reinvent themselves with the intent to remain sustainable. In this scenario, innovation, and agility to adapt to new market requirements are paramount. For the promotion of such characteristics, purchasing management becomes essential as it allows corporations a strategical performance to achieve the goals. Accordingly, the purpose of this study, that is exploratory and descriptive, is to evidence a literary portfolio regarding the topic of purchasing management and to indicate important variables in accordance with the boundaries posed by researchers. For this, we used the Knowledge Development Process - Constructivist (Proknow-C). It was possible to obtain twenty publications on the subject and with this material it was concluded that: the Journal of Purchasing and Supply Management was the journal with the highest number of related publications; the article titled Supply Management Orientation and Supplier / buyer performance, was significantly evidenced as a result of the number of citations, the authors who had the most articles in the bibliography portfolio, are Sánchez-Rodríguez, E. Hartmann and R. Moser and the authors of the literary portfolio that were most cited were D. A. Collier and D. D. Wilson, the keywords most utilized in the portfolio were performance, followed by purchasing and management.
\end{abstract}

Keywords: Purchasing. Performance Evaluation. Organizations. Proknow-C. 


\section{INTRODUÇÃO}

Até a década de 60, as empresas se assentavam em um ambiente estável cujas condições de trabalho permaneciam inalteradas por anos, sem necessidade de mudanças impactantes. Essa estabilidade estava presente em todos os níveis das empresas, desde níveis mais estratégicos até gerenciais de departamentos. O departamento de compras, por exemplo, tinha o propósito de garantir a disponibilidade de materiais no longo prazo e assim assegurar a continuidade dos negócios. Tal departamento tinha como rotina a negociação com uma rede de fornecedores consolidada (KRALJIC, 1983). Sendo assim, a função desse departamento se restringia a abastecer a organização com preço e qualidade vantajosos.

Atualmente, as funções de preço e qualidade adicionaram-se às dimensões, tais como: agilidade, flexibilidade, inovação, comprometimento social e ambiental. Nesse cenário, gerenciar os suprimentos passa a ser essencial para que empresas se mantenham competitivas, pois é a gestão de suprimentos que mune os diversos setores com recursos, de maneira estratégica, para o alcance do sucesso das organizações.

Nesse sentido, o departamento de compras passa a ter funções mais abrangentes, tais como: obter mercadorias e serviços nas quantidades e qualidade necessários; obter mercadorias e serviços ao menor custo; garantir o melhor serviço possível e pronta entrega por parte do fornecedor; desenvolver e manter boas relações com os fornecedores e desenvolver fornecedores potenciais. Para dar suporte a essas e outras atividades, a avaliação de desempenho emerge como um instrumento que permite conhecer o que é importante para o contexto; mensurar esses aspectos, integrá-los e gerar ações para o aperfeiçoamento (CHAVES et al, 2013).

Esse contexto motivou os pesquisadores deste trabalho a buscarem informações sobre o tema gestão de suprimentos nas organizações. Emergindo assim a pergunta de pesquisa: "Como evidenciar e analisar as informações de publicações científicas referentes ao tema "gestão de suprimentos" quando analisado através do olhar de seu desempenho?"

Nesse sentido, o objetivo geral para responder à pergunta de pesquisa passa a ser: selecionar as publicações de destaque referentes ao tema "gestão de suprimentos" quando analisado através do olhar de seu desempenho e evidenciar para este portfólio bibliográfico os artigos, autores, periódicos e palavras-chave mais recorrentes.

Para tanto, foram utilizados os seguintes objetivos específicos:

(i) Selecionar um Portfólio Bibliográfico (PB) composto por artigos internacionais referentes ao assunto estabelecido conforme a percepção dos pesquisadores; e 
(ii) Destacar os principais periódicos, artigos, autores e palavras-chave.

Como instrumento de intervenção, utilizar-se-á o processo ProKnow-C (Knowledge Development Process - Constructivist), de modo a selecionar artigos relevantes em um portfólio internacional e identificar características dessas publicações para informar à comunidade acadêmica e praticante sobre o tema de interesse.

O presente artigo tem um enfoque bibliométrico que propicia uma compreensão melhor sobre determinado campo de pesquisa (ARAÚJO; ALVARENGA, 2011), no caso deste artigo a gestão de suprimentos.

Este artigo está estruturado em cinco seções, além desta introdutória, apresentando em seguida a metodologia da pesquisa utilizada, referencial teórico, resultados e considerações finais.

\section{METODOLOGIA}

\subsection{Enquadramento Metodológico}

A presente pesquisa se sustenta no enquadramento metodológico como proposto por Ensslin, Ensslin e Pinto (2013) e Tasca et al., (2010),

Pode-se afirmar que é uma pesquisa descritiva, pois relata os fenômenos da realidade estudada. De acordo com Gil (1999), este tipo de pesquisa objetiva descrever as características de determinada população, fenômeno ou estabelecimento de relações entre as variáveis. Nesse artigo descrevem-se as características de um portfólio bibliográfico. Também é considerada exploratória, pois, conforme Gil (1999), promove reflexão do assunto investigado, com a construção de hipóteses, permitindo maior familiaridade com o problema e geração de conhecimento.

Em relação à abordagem, classifica-se como uma pesquisa qualitativa-quantitativa, conforme exposto por Richardson (1999). Qualitativa porque a percepção dos decisores mantiveram-se presentes para a construção de um portfólio bibliográfico. Quantitativa porque se analisaram variáveis numéricas do portfólio.

Considerando-se os procedimentos técnicos, esta pesquisa enquadra-se na classificação de Gil (1999) como uma pesquisa bibliográfica, por ser elaborada a partir de material já publicado, neste caso artigos de periódicos. 


\subsection{Instrumento de intervenção}

De acordo com Rosa et al., (2012), a identificação de artigos relevantes que geram conhecimento e suportam a realização de pesquisas científicas tem se tornado muito complexa, devido à grande quantidade de informações disponíveis nas literaturas nacional e internacional, disponíveis em variadas fontes de pesquisa.

Diante disto, optou-se por utilizar neste estudo o processo Knowledge Development Process-Constructivist (Proknow-C). De acordo com Ensslin, Ensslin e Pinto (2013), este modelo foi concebido no Laboratório de Metodologias Multicritério em Apoio à Decisão (LabMCDA), vinculado ao Departamento de Engenharia de Produção e Sistemas da Universidade Federal de Santa Catarina, o qual desde 1994 investiga o tema Avaliação de Desempenho Organizacional como instrumento de Apoio à Decisão, por meio da metodologia Multicritério em Apoio à Decisão - Construtivista (MCDA-C), em nível teórico e prático. Entretanto, o LabMCDA percebeu que os estudos que informavam a revisão do estado da arte de suas publicações poderiam ser questionados quanto ao alinhamento e à relevância do conteúdo, fato que levou à constatação da ausência de um processo estruturado para a seleção e análise da literatura científica.

A partir de 2005, foi criada pelo LabMCDA uma linha de pesquisa para suprir essa lacuna, com o desenvolvimento de um processo que pudesse realizar a busca com a amplitude delimitada. Em 2010 surgiram as primeiras publicações e, posteriormente, os integrantes do LabMCDA atribuíram o nome de Knowledge Development Process-Construtivist (ProKnow$C)$ ao processo para atender às solicitações de registro e de originalidade. Segundo Ensslin, Ensslin e Pinto (2013), em 2012 o ProKnow-C já contava com diversas publicações em periódicos nacionais e internacionais, consolidando-se como processo para mapeamento do conhecimento, segundo as delimitações, percepções do tema e motivações do pesquisador.

O processo ProKnow-C é constituído de quatro etapas: Seleção de Portfólio Bibliográfico, análise bibliométrica, análise sistêmica e formulação da pergunta de pesquisa. Neste estudo foram desenvolvidas duas etapas do processo: a seleção de um portfólio bibliográfico, que tem por objetivo reunir um conjunto restrito de publicações com destaque científico, alinhadas a um determinado tema, de acordo com a percepção e delimitações de um pesquisador específico, a outra etapa considerada no presente trabalho foi a Bibliometria, a qual segundo Vaz et al. (2012) consiste no processo de evidenciação quantitativa dos dados estatísticos de um conjunto definido de artigos para a gestão da informação e do 
conhecimento científico de um dado assunto, com foco de observação nas publicações, autores, citações, periódicos e bancos de dados.

\section{REFERENCIAL TEÓRICO}

Nos últimos anos, o percentual referente ao volume de compras, com relação ao faturamento total da empresa, tem aumentado consideravelmente (SCHIELE, 2007). Nesse sentido, um desempenho melhor da função de compras pode contribuir significativamente para o desempenho global de uma organização (SANCHEZ-RODRIGUEZ et. al, 2005). Dentre os fatores relacionados à gestão de suprimentos, encontram-se na literatura estudos abrangendo diferentes aspectos que podem impactar positivamente no resultado na organização.

De acordo com Pohl e Foerstl (2007), o nível de integração e alinhamento entre a estratégia de suprimentos com os objetivos corporativos é um fator determinante considerando o impacto da função de compras no sucesso global da empresa. Nesse cenário, os autores objetivaram avaliar como os sistemas de medição de desempenho de compras devem ser projetados para permitir o alinhamento bem-sucedido entre as práticas de compras e a estratégia da organização. Para esses autores existem cinco categorias de papéis que a medição de desempenho de compras deve preencher: (a) Gestão estratégica; (b) Medidas de desempenho; (c) Influência comportamental; (d) Aprendizado e desenvolvimento e; (e) Comunicação. Nesse sentido, os autores supracitados desenvolveram as cinco proposições sobre como os sistemas de medição de desempenho de compras (PPMS) devem ser projetados para facilitar a integração estratégica, na tentativa de validar o que vinha sido abordado pela literatura. Isso foi feito por meio de 4 estudos de casos em empresas alemãs de diferentes setores. Concluiu-se com os estudos de caso que as dimensões aplicadas nos sistemas de medição de desempenho estavam alinhadas com a estratégia de suprimentos e refletiam os objetivos inerentes da estratégia da corporação. Porém, nem todos os objetivos estratégicos são traduzidos em medidas de desempenho. Os autores sugerem que sejam incorporados nas medidas de desempenho indicadores não financeiros.

Estudos anteriores de Shin, Collier e Wilson (2000) afirmam no final do século XX que acadêmicos e profissionais concordaram que a excelência na gestão do fornecimento resulta em melhor qualidade, serviço ao cliente e desempenho do canal. Os autores realizaram uma pesquisa com 176 indústrias automotivas americanas, com o objetivo de testar o impacto de uma orientação à gestão do fornecimento, a qual chamam Supply Management Orientation 
(SMO), voltada à avaliação de desempenho dos fornecedores e compradores. Foi constatado que melhorias na orientação da gestão do fornecimento são estatisticamente mais significativas se relacionadas à entrega e qualidade do que a custo e flexibilidade. Detectou-se também que os melhores resultados em relação ao indicador custo foram obtidos pelos fornecedores que estavam voltados à melhoria de entrega e qualidade, e não pelos fornecedores que estavam voltados à redução de custo. Por fim, os autores concluíram que melhorias na SMO são capazes de potencializar o desempenho tanto dos fornecedores como dos compradores, o que significa uma situação de "ganha-ganha" para cadeia de abastecimento.

\section{RESULTADOS E DISCUSSÕES}

Nesta seção apresentam-se os resultados da pesquisa e seleção de artigos utilizando o ProKnow-C para o contexto de avaliação de produção científica para (a) compor um portfólio bibliográfico e (b) realizar a análise bibliométrica do portfólio bibliográfico.

\subsection{Seleção do Portfólio Bibliográfico}

O objetivo desta etapa é selecionar um conjunto restrito de publicações com reconhecimento e destaque científico, alinhadas com o tema "gestão de suprimentos" quando analisado através do olhar de seu desempenho, de acordo com a percepção e delimitações dos pesquisadores. Para a seleção do Portfólio Bibliográfico - PB - realizaram-se as seguintes fases: 1) Seleção do banco bruto de artigos; 2) Filtro quanto à redundância, título e reconhecimento científico; 3) Filtro do banco bruto de artigos não repetidos e com títulos alinhados; e 4) Filtro quanto ao alinhamento integral do artigo. (Tasca et al., 2010; Lacerda et al., 2011; Bortoluzzi et al., 2011; Rosa et al., 2012; Ensslin et al., 2013). Tais fases estão previstas no ProKnow-C e apresentadas nos meses de março e abril de 2013.

\subsubsection{Seleção do banco de artigos brutos}

O ProKnow-C propõe para a busca de artigos científicos em base de dados, inicialmente, definir um conjunto de palavras-chave que, segundo a percepção do pesquisador, represente o assunto tema. Para assegurar a representatividade do tema, o ProKnow-C inicia instigando os pesquisadores a definir as dimensões necessárias e 
suficientes para definir o tema, para este estudo, denotados como eixos de pesquisa. Desta forma, na primeira etapa, primeiramente definiram-se os eixos de pesquisa, para em seguida determinar as palavras-chave em língua inglesa a serem consultadas nas bases de dados. A Figura 1 apresenta as atividades necessárias para seleção do banco bruto de artigos.

Figura 1 - Etapa da Fase de Seleção do Banco Bruto de Artigos para compor o Portfólio Bibliográfico.

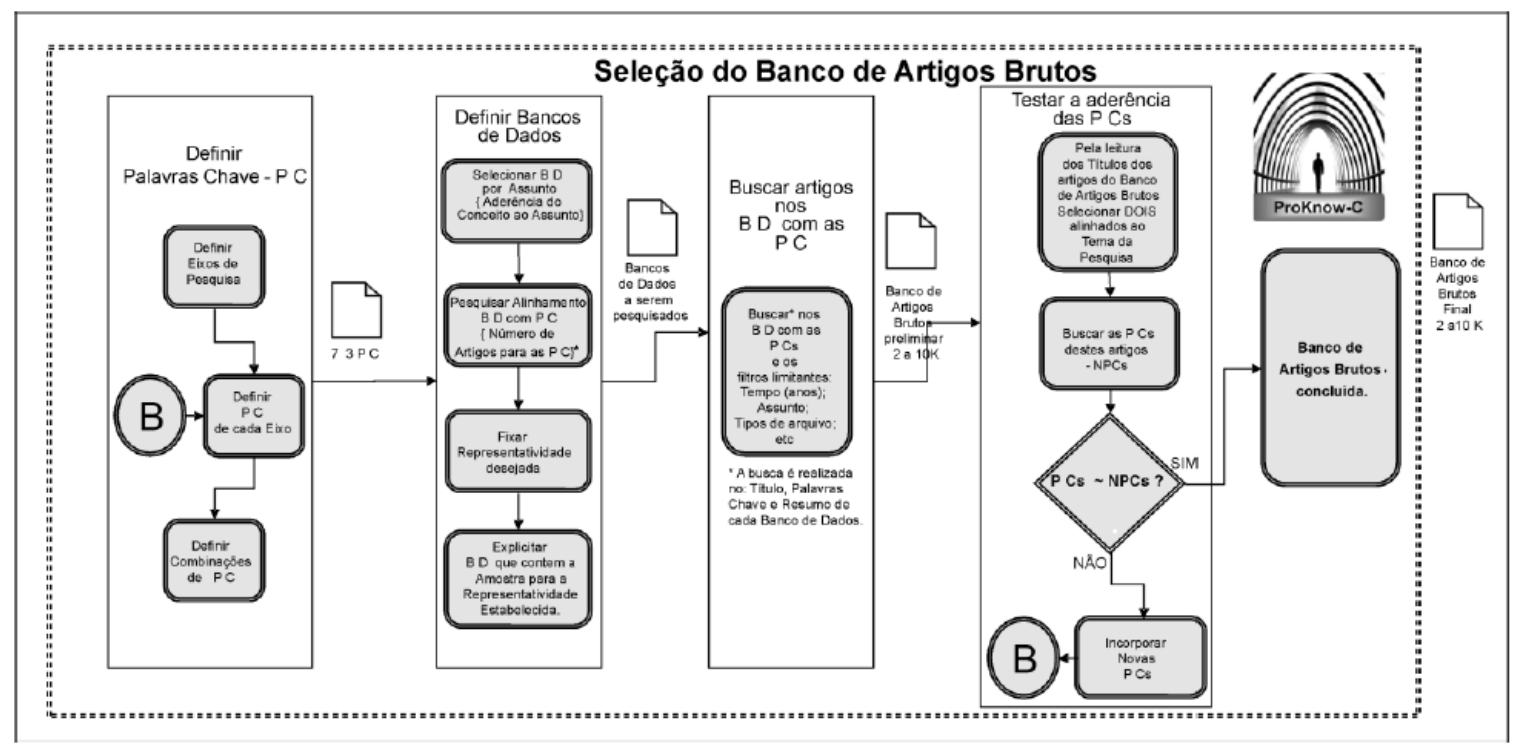

Fonte: Tasca et. al. (2010).

São demonstradas na Figura 1, quatro subetapas necessárias, as quais serão detalhadas no decorrer deste trabalho.

\subsubsection{Definição das palavras-chave}

Para iniciar essa fase foram criados os dois eixos de pesquisa que representam a percepção dos pesquisadores referente ao tema. Cada eixo possui um conjunto de palavras que o representa, os eixos são:

(i) Eixo - Avaliação de Desempenho:
a. Performance Measurement
b. Performance Evaluation
c. Performance Appraisal
d. Performance Assessment
e. Performance Management

(ii) Eixo-Suprimentos:
a. Supply*
b. Purchas* 

c. Procurement
d. Buying
e. Acquisition

O asterisco foi utilizado após o radical das palavras com o intuito de que a pesquisa alcançasse as variações verbais ou substantivas das palavras. Assim, chegou-se a 25 combinações possíveis com o cruzamento dos eixos que foram utilizados como norteadores na pesquisa.

\subsubsection{Definição dos bancos de dados}

De posse das combinações de palavras-chave, é necessária a definição das bases de dados que serão utilizadas para realização da pesquisa. $\mathrm{O}$ estudo foi baseado no Portal de Periódicos da CAPES, a qual possui conteúdo científico composto por 193 bases de diferentes áreas de conhecimento. Foram analisadas relevantes para esta pesquisa as bases das áreas Multidisciplinares, Engenharias e Ciências Sociais Aplicadas. Dentre essas, foram selecionadas 6 bases, a saber: SciVerse Scopus, Wiley, Web of Knowledge - ISI, Engineering Village, Oxford e Proquest.

\subsubsection{Busca de artigos nos bancos de dados com as palavras-chave}

O processo de busca nas bases de periódicos, a partir da utilização das palavras-chave definidas pelos autores, foi realizado entre os dias 02 e 07 de abril de 2013. Ao utilizar-se as palavras-chave anteriormente definidas e a data de publicação após o ano 2000, a busca nas 6 bases de dados retornou um volume de 6.459 artigos conforme ilustrado Figura . Este montante compôs o banco bruto de artigos. Para a reunião dos trabalhos e composição do Banco de Artigos, foi utilizado o Endnote X3 como gerenciador bibliográfico. 
Figura 2 - Resultado da busca nas bases de dados.

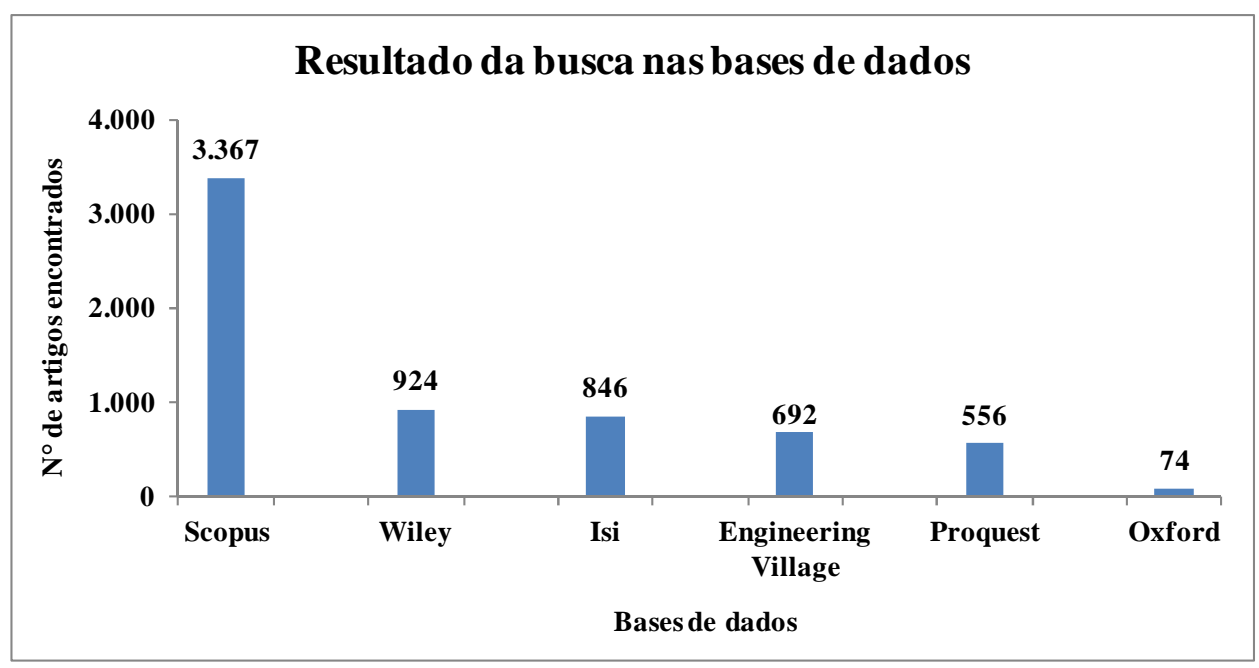

Fonte: Autores.

Observa-se no gráfico da Figura 2 que, dentre os 6.459 artigos disponíveis em 6 bases de dados, a maioria dos artigos foram obtidos pela base Scopus, a qual representa $52 \%$ do conteúdo do Banco Bruto de Artigos. Para testar se as palavras-chave que retornaram essa pesquisa são congruentes ao tema, recorre-se ao teste de aderência das palavras-chave, o qual é apresentado a seguir.

\subsubsection{Teste da aderência das palavras-chave}

Pela leitura dos títulos dos artigos presentes no banco de artigos brutos, foram selecionados 6 alinhados ao tema da pesquisa, com o objetivo de identificar as palavras-chave desses artigos e validar a necessidade de se incluir novas palavras-chave. Nesta análise, percebeu-se como suficiente a utilização de combinações já destacadas inicialmente. Outras palavras-chave encontradas não estavam alinhadas ao tema definido pelos pesquisadores. Concluiu-se então que não seria necessária a inclusão de novas palavras-chave.

\subsubsection{Filtragem do banco de artigos}

Nesta etapa, conforme ilustrado nas Figura, Figura 4 e Figura 5 são realizados quatro tipos de filtros, de acordo com a sequência abaixo. 


\subsubsection{Filtro quanto a redundância}

O primeiro de artigos no ProKnow-C é o Filtro quanto à redundância, que tem como propósito eliminar arquivos repetidos ou que foram publicados em periódicos conforme se observa na Figura 3.

Figura 3 - Etapa da Fase de Filtragem do Banco de Artigos.

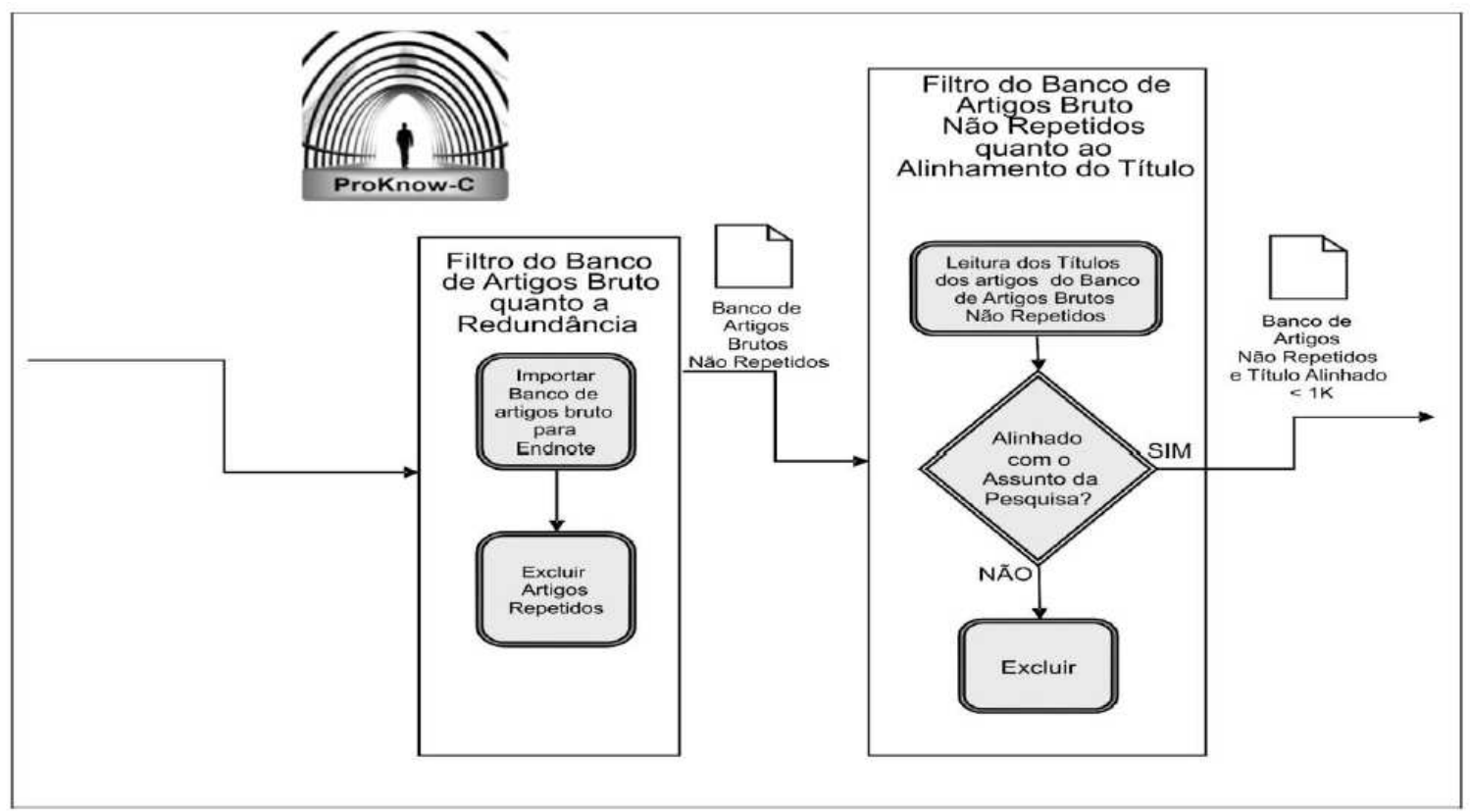

Fonte: Tasca et. al. (2010).

Com a utilização do gerenciador bibliográfico Endnote $X 3$, foi possível identificar 1.381 referências, as quais abrangiam artigos repetidos, publicações em conferências, livros, capítulos de livros, patentes, séries, que acabaram sendo importadas mesmo com os filtros estabelecidos nas bases de dados. Estas foram excluídas, de modo a restar no banco, um total de 5.078 artigos.

\subsubsection{Filtro quanto ao alinhamento do título ao tema de pesquisa}

Neste momento foi realizada a leitura dos 5.078 títulos dos artigos, permitindo aos pesquisadores selecionarem sob seu critério de avaliação, 253 artigos com o título alinhado ao tema de pesquisa. 


\subsubsection{Filtro quanto ao reconhecimento científico}

Nesta etapa, consulta-se no site do Google Acadêmico a quantidade de citações de cada um dos 253 artigos com títulos alinhados ao tema da pesquisa. Para esta atividade foram utilizados os softwares Zotero e Excel, os quais auxiliaram na identificação de que 105 artigos possuíam mais de 12 citações, os quais foram incorporados ao Repositório K. Para seleção deste conjunto de artigos foram fixadas como representativas $96 \%$ das citações de todas 253 referências. Os outros 148 artigos, com menos de 12 citações, foram separados no Repositório $\mathrm{P}$, classificados como banco de artigos com títulos alinhados com reconhecimento científico ainda não confirmado. Essa parte do processo pode ser visualizada na Figura 4.

Em seguida, realizou-se a leitura dos resumos dos 105 artigos presentes no Repositório $\mathrm{K}$ para verificar o alinhamento do conteúdo com o tema. Destes, 14 se enquadraram ao tema e foram classificados como integrantes de um novo repositório, chamado aqui de Repositório A - Banco de Artigos com título, resumo alinhado e reconhecimento científico. A partir daí, identificaram-se 33 autores destes artigos, formando assim o Banco de Autores (BA).

Figura 4 - Etapa da Fase de Filtragem do Banco de Artigos.

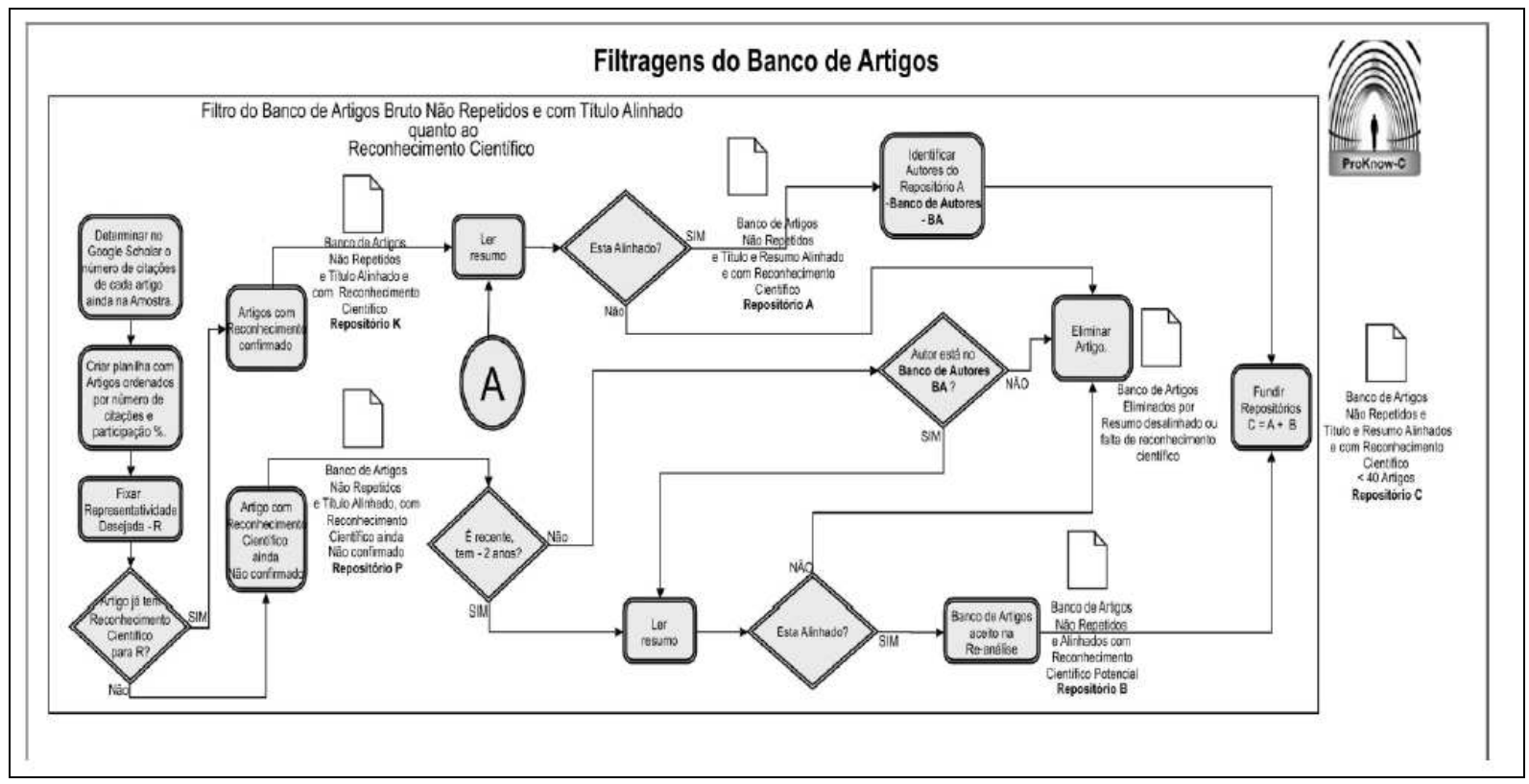

Fonte: Tasca et. al. (2010).

Ainda nesta etapa, analisam-se os artigos do repositório $\mathrm{P}$ a fim de identificar outros artigos com potencial para compor o portfólio bibliográfico. Para tal análise consideram artigos com 2 anos ou menos que não tiveram tempo suficiente para serem citados e artigos 
dos autores do BA que já possuem artigos relevantes ao assunto. Dos 131 artigos do repositório, 63 foram publicados após janeiro de 2011 e nenhum possuía autor vinculado ao BA. Assim, fez-se uma leitura dos resumos dos 63 artigos com o intuito de identificar o alinhamento com o tema. Nessa fase, 9 artigos foram considerados pertencentes ao Repositório B - Banco de Artigos com título, resumo alinhado e reconhecimento científico potencial. Ao fundir os artigos do Repositório A e B, forma-se então o Repositório C, composto por 23 artigos.

\subsubsection{Filtro quanto ao alinhamento do artigo integral ao tema de pesquisa}

Nesta fase final de filtragem, checou-se primeiramente a disponibilidade gratuita dos 23 artigos do Repositório C - Banco de Artigos com título, resumo alinhado e reconhecimento científico confirmado ou potencial. Desses, 2 artigos não estavam disponíveis gratuitamente. Por fim, foi realizada leitura integral dos 21 artigos restantes, detectando que 4 deles não estavam alinhados com o tema de pesquisa. Este processo é visualizado na Figura 5.

Figura 5 - Etapa da Fase de Filtragem do Banco de Artigos.



Fonte: Tasca et. al. (2010).

Sendo assim, 17 referências representaram a relação de artigos do portfólio bibliográfico primário. Em seguida, realiza-se um teste para verificar se há potenciais artigos ao Portfólio Bibliográfico que não foram considerados até esta etapa do processo. 


\subsubsection{Teste de Representatividade do Portfólio Bibliográfico}

Nesta etapa é possível avaliar se algum artigo presente nas referências bibliográficas do portfólio selecionado pode ser adicionado ao próprio portfólio. Para tanto, é necessário seguir um processo estruturado, similar ao já realizado para seleção do Portfólio Bibliográfico. Desta forma avaliaram-se todas as 316 referencias representadas por artigos publicados em periódicos a partir do ano 2000. Novamente utilizou-se o gerenciador bibliográfico Endnote X3 e com o auxílio dos softwares Zotero e Excel, identificou-se que 74 artigos possuíam mais de 222 citações, o que representa $80 \%$ do total das citações. Com a leitura destas referências, identificou-se que 3 artigos estavam integralmente alinhados ao tema de pesquisa e, portanto, foram incorporados ao Portfólio Bibliográfico Primário. Sendo assim, o Portfólio Bibliográfico para o tema gestão de suprimentos passou a ser composto por 20 artigos. Os quais podem ser visualizados no Quadro 1.

\section{QUADRO 1- ARTIGOS SELECIONADOS PARA O PORTFÓLIO}

\begin{tabular}{|c|c|c|c|}
\hline Autor & Título & Ano & Citações \\
\hline $\begin{array}{l}\text { H. Shin, D. A. Collier and } \\
\text { D. D. Wilson }\end{array}$ & $\begin{array}{l}\text { Supply management orientation and supplier/buyer } \\
\text { performance }\end{array}$ & 2000 & 500 \\
\hline $\begin{array}{l}\text { I. J. Chen, A. Paulraj and A. } \\
\text { A. Lado }\end{array}$ & $\begin{array}{l}\text { Strategic purchasing, supply management, and firm } \\
\text { performance }\end{array}$ & 2004 & 348 \\
\hline $\begin{array}{l}\text { T. Y. Choi and D. R. } \\
\text { Krause }\end{array}$ & $\begin{array}{l}\text { The supply base and its complexity: Implications for } \\
\text { transaction costs, risks, responsiveness, and } \\
\text { innovation }\end{array}$ & 2006 & 241 \\
\hline $\begin{array}{l}\text { L. Easton, D. J. Murphy and } \\
\text { J. N. Pearson }\end{array}$ & $\begin{array}{l}\text { Purchasing performance evaluation: With data } \\
\text { envelopment analysis }\end{array}$ & 2002 & 78 \\
\hline $\begin{array}{l}\text { R. A. M. Narasimhan, J. J. } \\
\text { Aram, Carter and R. Joseph }\end{array}$ & $\begin{array}{l}\text { An empirical examination of the underlying } \\
\text { dimensions of purchasing competence }\end{array}$ & 2001 & 70 \\
\hline $\begin{array}{l}\text { S. M. Wagner and L. } \\
\text { Kaufmann }\end{array}$ & $\begin{array}{l}\text { Overcoming the main barriers in initiating and using } \\
\text { purchasing-BSCs }\end{array}$ & 2004 & 42 \\
\hline $\begin{array}{l}\text { C. Baier, E. V. I. Hartmann } \\
\text { and R. Moser }\end{array}$ & $\begin{array}{l}\text { Strategic alignment and purchasing efficacy: an } \\
\text { exploratory analysis of their impact on financial } \\
\text { performance }\end{array}$ & 2008 & 42 \\
\hline H. Schiele & $\begin{array}{l}\text { Supply-management maturity, cost savings and } \\
\text { purchasing absorptive capacity: Testing the } \\
\text { procurement-performance link }\end{array}$ & 2007 & 40 \\
\hline R. O. Large and T. Koenig & $\begin{array}{l}\text { A gap model of purchasing's internal service quality: } \\
\text { Concept, case study and internal survey }\end{array}$ & 2009 & 27 \\
\hline $\begin{array}{l}\text { J. R. Carter, L. R. Smeltzer } \\
\text { and R. Narasimhan }\end{array}$ & $\begin{array}{l}\text { Human Resource Management within Purchasing } \\
\text { Management: Its Relationship to Total Quality } \\
\text { Management Success }\end{array}$ & 2000 & 27 \\
\hline $\begin{array}{l}\text { C. Sánchez-Rodríguez, Á. } \\
\text { R. Martínez-Lorente and J. } \\
\text { G. Clavel }\end{array}$ & $\begin{array}{l}\text { Benchmarking in the purchasing function and its } \\
\text { impact on purchasing and business performance }\end{array}$ & 2003 & 21 \\
\hline $\begin{array}{l}\text { L. D. Fredendall, C. D. } \\
\text { Hopkins and A. Bhonsle }\end{array}$ & $\begin{array}{l}\text { Purchasing's Internal Service Performance: Critical } \\
\text { External and Internal Determinants }\end{array}$ & 2005 & 17 \\
\hline
\end{tabular}




\begin{tabular}{|l|l|l|l|}
\hline $\begin{array}{l}\text { D. Hemsworth, C. Sánchez- } \\
\text { Rodríguez and B. Bidgood }\end{array}$ & $\begin{array}{l}\text { Determining the impact of quality management } \\
\text { practices and purchasing-related information } \\
\text { systems on purchasing performance: A structural } \\
\text { model }\end{array}$ & 2005 & 15 \\
\hline H. Saranga and R. Moser & $\begin{array}{l}\text { Performance evaluation of purchasing and supply } \\
\text { management using value chain DEA approach }\end{array}$ & 2010 & 13 \\
\hline M. Pohl and K. Foerstl & $\begin{array}{l}\text { Achieving purchasing competence through } \\
\text { purchasing performance measurement system } \\
\text { design-A multiple-case study analysis }\end{array}$ & 2011 & 6 \\
\hline $\begin{array}{l}\text { S. H. Elgazzar, N. S. Tipi, } \\
\text { N. J. Hubbard and D. Z. } \\
\text { Leach }\end{array}$ & $\begin{array}{l}\text { Linking supply chain processes' performance to a } \\
\text { company's financial strategic objectives }\end{array}$ & 2012 & 3 \\
\hline $\begin{array}{l}\text { E. Hartmann, D. Kerkfeld } \\
\text { and M. Henke }\end{array}$ & $\begin{array}{l}\text { Top and bottom-line relevance of purchasing and } \\
\text { supply management }\end{array}$ & 2012 & 2 \\
\hline $\begin{array}{l}\text { L. Chen, S. M. Gilbert and } \\
\text { X. Xu }\end{array}$ & $\begin{array}{l}\text { The role of revenue-focused managerial } \\
\text { performance measures in supply chain coordination }\end{array}$ & 2012 & 1 \\
\hline $\begin{array}{l}\text { H. L. Chu, C. C. Cho and S. } \\
\text { Z. Liu }\end{array}$ & $\begin{array}{l}\text { Effects of unbalanced incentive measures on buyer } \\
\text { performance - an empirical study in Taiwan }\end{array}$ & 2011 & 0 \\
\hline $\begin{array}{l}\text { D. Kern, R. Moser, N. } \\
\text { Sundaresan and E. } \\
\text { Hartmann }\end{array}$ & $\begin{array}{l}\text { Purchasing competence: A stakeholder-based } \\
\text { framework for Chief Purchasing Officers }\end{array}$ & 2011 & 0 \\
\hline
\end{tabular}

A partir dos artigos que compõem o Portfólio Bibliográfico e suas referências, procede-se a uma análise bibliométrica, com vistas a realizar o mapeamento do tema "gestão de suprimentos" quando analisado através do olhar de seu desempenho para evidenciar para este portfólio bibliográfico os artigos, autores, periódicos e palavras-chave mais recorrentes.

\subsection{Análise Bibliométrica}

Nesta etapa, é realizada uma análise dos artigos do Portfólio Bibliográfico e respectivas referências bibliográficas, com o objetivo de quantificar as informações existentes e fornecer as características destas publicações, considerando grau de relevância dos periódicos, reconhecimento científico dos artigos, autores de maior destaque e palavras-chave mais utilizadas. Esta análise foi realizada nos meses de abril e maio de 2013.

\subsubsection{Relevância dos periódicos}

Com relação à análise realizada sobre a relevância dos periódicos do portfólio bibliográfico e de suas das referências, é possível visualizar na Figura , que, dentre as 20 publicações do Portfólio, 5 encontram-se no Journal of Purchasing and Supply Management. 
Figura 6 - Relevância dos periódicos.



Fonte: Autores.

O fato de o periódico Journal of Purchasing and Supply Management ter o maior número de artigos no portfólio significa que os pesquisadores da pesquisa entenderam que esses artigos eram alinhados ao tema. Portanto, o periódico é considerado alinhado ao tema dessa pesquisa. Isso se confirma no delimitado escopo da revista que aborda compras e cadeia de suprimentos.

Já o periódico que se destaca nas referências do portfólio, é o Journal of Operations Management, com 15 artigos. Isso pode ser explicado devido ao fato de que o periódico é indexado nas bases IsiKnowledge e Scopus e tem fatores de impacto JCR e SJR de 4.4 e 5 respectivamente, o que contribui para que o periódico receba citações nas referências do PB, além de seu amplo escopo ao abordar gestão de operações de uma maneira geral.

O Journal of Supply Chain Management possui 3 artigos do Portfólio e 2 nas referências e, por isso, é também considerado um destaque dentre os periódicos.

\subsubsection{Reconhecimento científico dos artigos}

É possível observar na Erro! Fonte de referência não encontrada., que o artigo do portfólio bibliográfico com maior reconhecimento é o Supply management orientation and supplier/buyer performance com 500 citações. Em seguida, encontra-se o Strategic purchasing, supply management, and firm performance com 348 citações, seguido por The supply base and its complexity: Implications for transaction costs, risks, responsiveness, and innovation com 241 citações. 
Cabe ressaltar que os 3 artigos mais citados, apresentados na Erro! Fonte de referência não encontrada., foram incluídos no portfólio na fase descrita na seção 4.1.3 Teste de Representatividade do Portfólio Bibliográfico.

Figura 7 - Relevância dos artigos no portfólio.

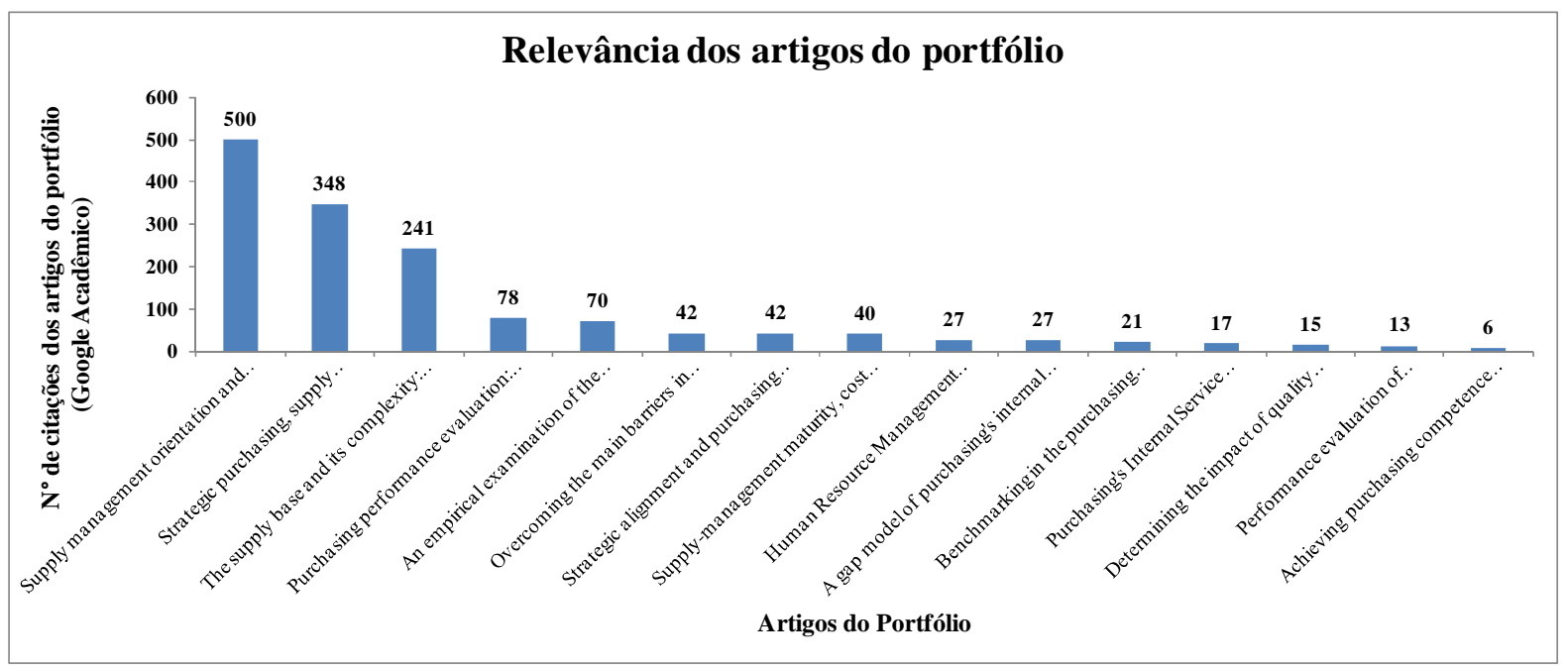

Fonte: Autores.

Observa-se que, dentre os artigos mais citados nas referências do portfólio, demonstrados na Figura 8 encontram-se trabalhos que envolvem o tema desta pesquisa de forma indireta, sem abranger especificamente os assuntos relacionados às palavras-chave utilizadas. Considerando a área de estudo, artigos relacionados a supply chain, são os mais presentes. Cabe salientar que, os três artigos mais citados são procedentes do periódico Journal of Operations Management, ou seja, o fato de o periódico estar indexados em 2 bases com um fator de impacto em destaque em ambas pode ter contribuído com o número de citação dos artigos do PB. Cabe ressaltar que o fator de impacto se refere ao número de citações do periódico e não do artigo (MATTOS; JOB, 2008), mas o fator de impacto de um periódico pode contribuir naturalmente com o aumento de citações de determinado artigo. 
Figura 8 - Relevância dos artigos nas referências do portfólio.

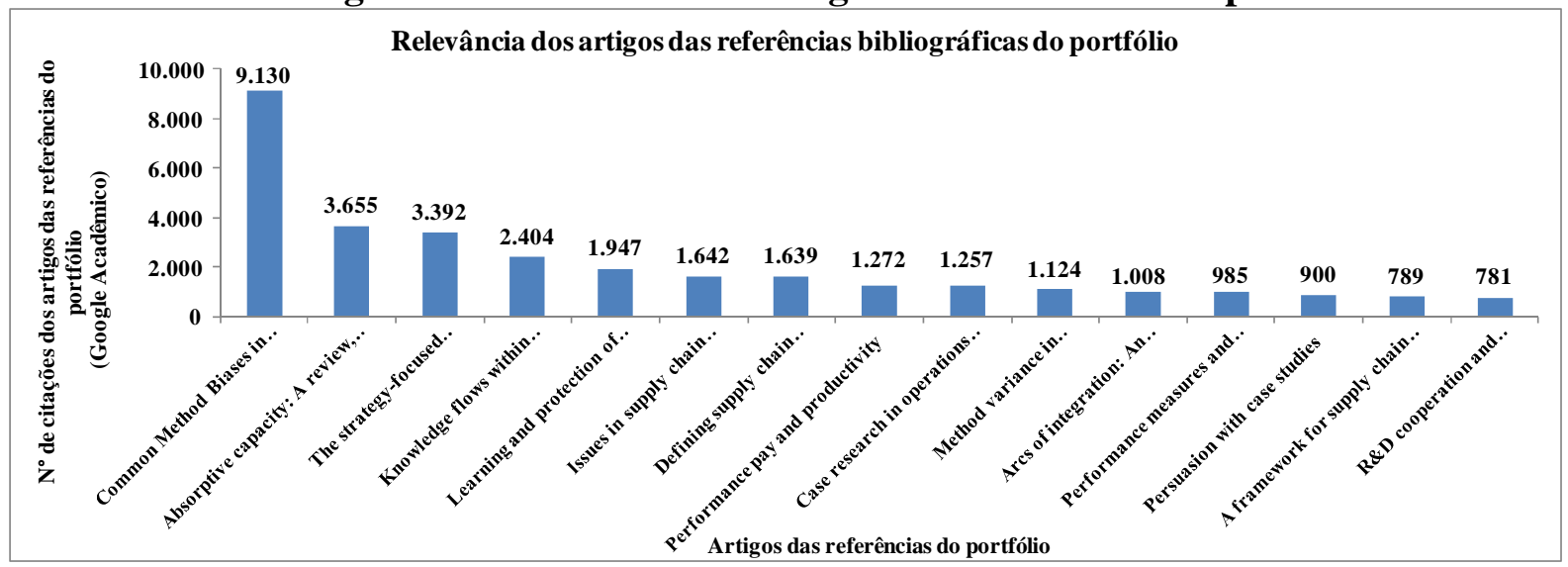

Fonte: Autores.

É possível visualizar que os artigos das referências do portfólio possuem um número de citações maior que o portfólio como pode ser observado nas Erro! Fonte de referência não encontrada. e Figura . Isso pode ser explicado pelo fato de que os artigos das referências que foram analisados na bibliometria não têm restrição temporal.

\subsubsection{Autores de maior destaque}

Dentre os 36 autores do portfólio bibliográfico, 3 autores, 2 publicações no portfólio, C. Sánchez-Rodríguez, E. Hartmann e R. Moser. Dentre os principais autores do portfólio, ressalta-se que 4 deles possuem destaque também nas referências do portfólio, que são D. A. Collier e D. D. Wilson com 2 publicações nas referências além de 1 publicação no portfólio, e T. Y. Choi e D. R. Krause com 1 publicação tanto no portfólio como nas referências. Na Figura 9 pode-se visualizar esta afirmação. 
Figura 9 - Autores mais relevantes.

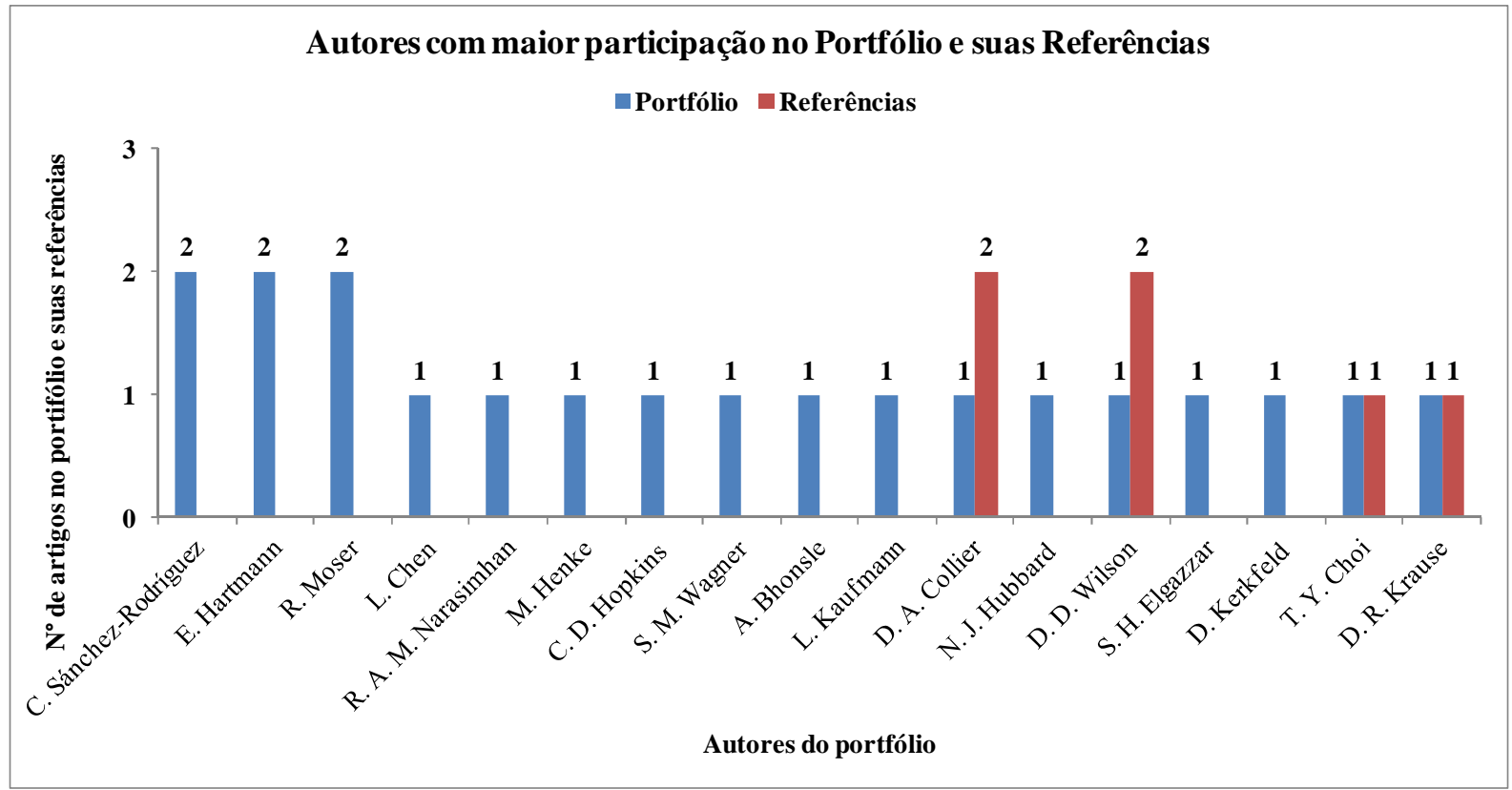

Fonte: Autores.

É possível identificar que, dentre os autores com maior participação no portfólio bibliográfico, não foram encontradas autocitações. Fato que ameniza distorções em análises.

\subsubsection{Palavras-Chave mais utilizadas}

Nesta seção, analisam o número de ocorrências das palavras-chave presentes no portfólio bibliográfico, em que destacam 8 , conforme se observa na Figura 0.

Figura 10 - Palavras-chave mais utilizadas.

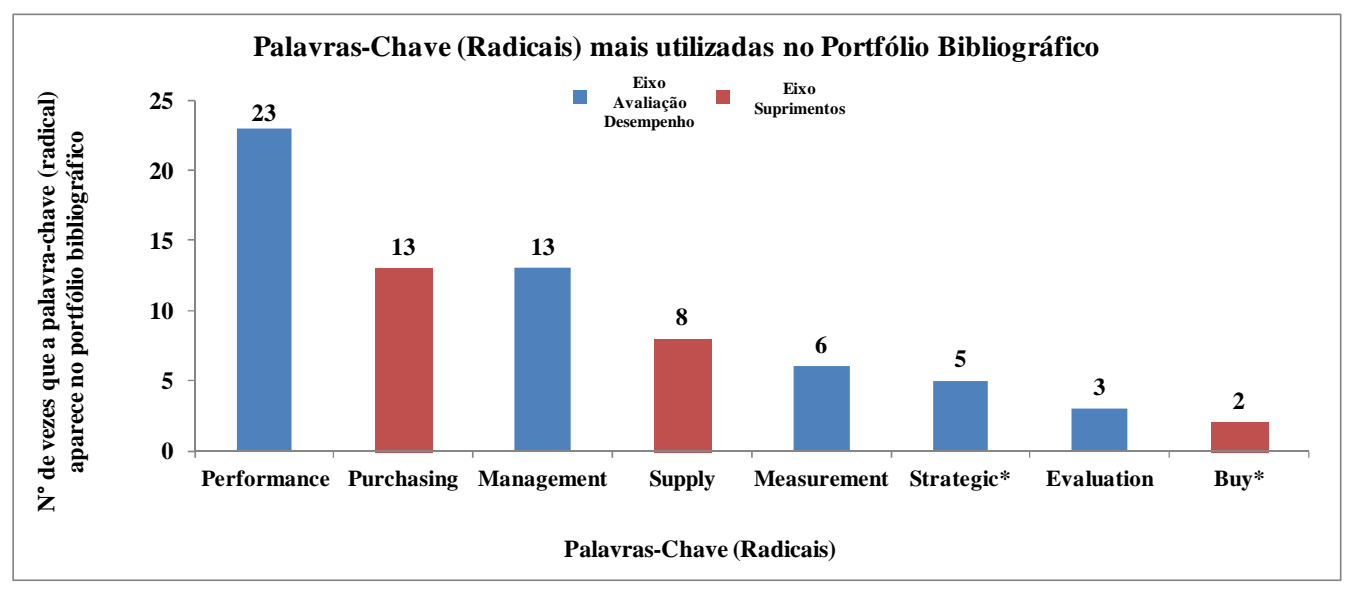

Fonte: Autores 
Observa-se que as palavras associadas ao tema avaliação de desempenho (demonstradas na figura pela cor azul) com maior representatividade são Performance, Management, Measurement, Strategic*, e Evaluation respectivamente. Já atreladas ao tema suprimentos (demonstradas na imagem sob a cor vermelha), pode-se identificar que Purchasing foi encontrada 13 vezes, seguida de Supply com 8 ocorrências e Buy* com 2 aparições.

\section{CONSIDERAÇÕES FINAIS}

A importância da função de compras no atendimento da estratégia organizacional motivou os pesquisadores deste trabalho a pesquisarem sobre o tema. Nesse sentido, o objetivo deste trabalho foi selecionar as publicações de destaque referentes ao tema "gestão de suprimentos" quando analisado através do olhar de seu desempenho e evidenciar para este portfólio bibliográfico os artigos, autores, periódicos e palavras-chave mais recorrentes de acordo com as percepções dos pesquisadores ao tema. Para tanto, utilizou-se o ProKnow-C (Knowledge Development Process - Constructivist) como instrumento de intervenção.

Primeiramente foi contemplado o objetivo específico “(i) Selecionar um Portfólio Bibliográfico (PB) composto por artigos internacionais referentes ao assunto, estabelecido conforme a percepção dos pesquisadores" na seção 4.1. O processo utilizado no presente estudo, foi originado com a busca em 6 bases de dados, que apontaram 6.459 artigos inicialmente. De acordo com a metodologia, foi selecionado sob a ótica dos pesquisadores, um portfólio bibliográfico composto de 20 artigos, alinhados à gestão de suprimentos, os quais podem ser identificados no Quadro 1.

Para atender ao objetivo específico “(ii) Elucidar os principais periódicos, artigos, autores e palavras-chaves" realizou-se uma análise bibliométrica que evidenciou que os periódicos que mais se destacaram foram Journal of Purchasing and Supply Management, Journal of Operations Management e Journal of Supply Chain Management. O trabalho de Shin, Collier e Wilson (2000) intitulado como Supply management orientation and supplier/buyer performance recebeu 500 citações e, portanto, destacou-se quanto ao número de citações. Os pesquisadores C. Sánchez-Rodríguez, E. Hartmann, R. Moser, D. A. Collier e D. D. Wilson obtiveram pelo menos 2 publicações no Portfólio Bibliográfico ou nas referências do Portfólio Bibliográfico. As palavras-chave mais presentes no Portfólio Bibliográfico foram Performance, Purchasing e Management. 
Cabe ressaltar alguns fatos observados pelos autores deste trabalho: O periódico Journal of Purchasing and Supply Management está indexado internacionalmente e tem o escopo alinhado à temática desta pesquisa. Por outro lado, o Journal of Operations Management, apesar de ter um escopo amplo, apresenta alguns artigos que também abordam o tema e que geralmente são mais referenciados pelo fato de o periódico apresentar alto fator de impacto nas bases em que está indexado. Isso condiz com o fato de o periódico apresentar os 3 artigos mais citados do Portfólio Bibliográfico composto de 20 artigos. Dentre os autores do Portfólio proposto, C. Sánchez-Rodríguez detém 2 artigos e aborda a relação entre as compras e o desempenho organizacional. E. Hartmann e R. Moser também possuem 2 artigos no Portfólio. E. Hartmann aborda a relevância entre compras e gestão de suprimentos. R. Moser aplica a análise envoltória de dados para avaliar a gestão de suprimentos e compras. E. Hartmann e R. Moser também apresentam um trabalho em conjunto que evidencia o impacto financeiro do alinhamento entre estratégia e eficácia de compras. Observa-se que todos os autores supracitados se dedicam a trabalhar com enfoque quantitativo relacionar variáveis e, assim, sustentar seus trabalhos.

As limitações desta pesquisa estão relacionadas ao limite temporal definido pelos autores, que consideraram publicações a partir do ano 2000 e ao escopo de abrangência do portal de periódicos da CAPES. Além disso, as delimitações estipuladas pelos pesquisadores estiveram presentes em diversas etapas do processo.

Para futuras pesquisas sugere-se uma análise quanto ao conteúdo do referencial levantado nesse trabalho. Sendo assim, será possível identificar oportunidades de aperfeiçoamento aos artigos presentes no Portfólio Bibliográfico e contribuir com esse campo de pesquisa.

\section{REFERÊNCIAS}

ARAÚJO, R. F.; ALVARENGA, L. A bibliometria na pesquisa científica da pós-graduação brasileira de 1987 a 2007. Encontros Bibli: revista eletrônica de biblioteconomia e ciência da informação, v. 16, n. 31, p. 51-70, 2011.

ARNOLD, J. R. Administração de materiais: uma introdução. 1. ed. São Paulo: Atlas, 1999.

ENSSLIN, L et al. Avaliação do Desempenho de Empresas Terceirizadas com o Uso da Metodologia Multicritério de Apoio à Decisão- Construtivista. Revista Pesquisa Operacional, v.30, n.1, p.125-152, 2010. 
ENSSLIN, L.; ENSSLIN, S. R.; PINTO, H. Processo de Investigação e Análise Bibliométrica: Avaliação da Qualidade dos Serviços Bancários. RAC, v. 17, n. 3, art. 4, pp. 325-349, 2013.

GIL, A. C. Métodos e Técnicas de Pesquisa Social. São Paulo: Atlas, 1999.

KRALJIC, P. Purchasing must become supply management. Harvard Business Review, v. 61, n. 5, p. 109-117, September-October 1983.

LAMBERT, D. M.; COOPER, M. C. Issues in supply chain management. Industrial Marketing Management, v. 29, p. 65 - 83, 2000.

MATTOS, A. M.; JOB, I. A produção científica brasileira no periódico Scientometrics de 1978 até 2006. Encontros Bibli: revista eletrônica de biblioteconomia e ciência da informação, v. 13, n. 26, p. 47-61, 2008.

RICHARDSON, R.J.; SOUSA PERES, J.A. Pesquisa social: métodos e técnicas. Atlas, 1999.

ROSA, F. S et al. Management environmental disclosure: a constructivist case. Management Decision, 50(6), 1117-1136, 2012.

SANCHEZ-RODRIGUEZ, C.; HEMSWORTH, D.; MARTINEZ-LORENTE, A. R. The effect of supplier development initiatives on purchasing performance: a structural model. Supply Chain Management: An International Journal 10 (4), 289-301, 2005.

SCHIELE H. Supply-management maturity, cost savings and purchasing absorptive capacity: Testing the procurement-performance link. Journal of Purchasing \& Supply Management 13, 274-293, 2007.

SILVA, E. H. D. R.; LIMA, E. P.; COSTA, S. E. G. Análise da produção acadêmica brasileira em medição de desempenho e modelos de gestão. Revista Gestão Industrial, v. 5, n. 3, p. 198-218, 2009.

SHIN, H; COLLIER D. A.; WILSON D. D. Supply management orientation and supplier/buyer performance. Benchmarking, v. 10, n. 5, p. 457-471. 2000.

TASCA, J. E. et al. An approach for selecting a theoretical framework for the evaluation of training programs. Journal of European Industrial Training, v. 34, n. 7, p. 631-655, 2010. 
VAZ, C. R et al. Avaliação de desempenho na gestão estratégica organizacional: seleção de um referencial teórico de pesquisa e análise bibliométrica. Revista Gestão Industrial, v. 8, n. 4, 2012.

\section{Como Referenciar este Artigo, conforme ABNT:}

ALEXANDRE, I. T; ENSSLIN, S. R; CHAVES, L. C; SILVA, J. M. D. Gestão de Suprimentos: Evidenciação e Análise das Publicações do Tema. Rev. FSA, Teresina, v.18, n. 9, art. 1, p. 3-25, set. 2021.

\begin{tabular}{|c|c|c|c|c|}
\hline Contribuição dos Autores & $\begin{array}{c}\text { I. } \mathbf{T} . \\
\text { Alexandre }\end{array}$ & $\begin{array}{c}\text { S. R. } \\
\text { Ensslin }\end{array}$ & $\begin{array}{c}\text { L. C. } \\
\text { Chaves }\end{array}$ & $\begin{array}{c}\text { J. M. D. } \\
\text { Silva }\end{array}$ \\
\hline 1) concepção e planejamento. & $\mathrm{X}$ & $\mathrm{X}$ & & \\
\hline 2) análise e interpretação dos dados. & $\mathrm{X}$ & $\mathrm{X}$ & $\mathrm{X}$ & \\
\hline 3) elaboração do rascunho ou na revisão crítica do conteúdo. & $\mathrm{X}$ & $\mathrm{X}$ & $\mathrm{X}$ & \\
\hline 4) participação na aprovação da versão final do manuscrito. & $\mathrm{X}$ & & $\mathrm{X}$ & $\mathrm{X}$ \\
\hline
\end{tabular}

